\title{
A New Biometric ID-Based Cryptography Protocol and Security Analysis Using Petri Nets
}

\author{
Dania Aljeaid \\ School of Science and Technology \\ Nottingham Trent University \\ Nottingham, United Kingdom \\ N0360890@ntu.ac.uk
}

\author{
Xiaoqi Ma \\ School of Science and Technology \\ Nottingham Trent University \\ Nottingham, United Kingdom \\ xiaoqi.ma@ntu.ac.uk
}

\author{
Caroline Langensiepen \\ School of Science and Technology \\ Nottingham Trent University \\ Nottingham, United Kingdom \\ caroline.langensiepen@ntu.ac.uk
}

\begin{abstract}
This paper presents a Petri net (PN) approach to modelling, simulating, and analysing the new protocol we have proposed. This new protocol is an enhanced authentication scheme based on a biometric verification mechanism and identity based cryptography. A formal approach like Petri nets allows one to represent cryptographic protocols. For the sake of simplicity, a complex PN model will not be discussed in this paper until all attacks are demonstrated and the model proved to be secure. This paper shows how Petri nets are used to model, analyse and detect flaws in our new protocol. First, our proposed protocol is modelled without an adversary, and then a generic adversary model is added to examine all possible adversary behaviours. Finally we demonstrate how Petri nets can be used to analyse security threats such as man-in-the-middle attack, reflection attack, and parallel session attack on this protocol.
\end{abstract}

Keywords- identity-based cryptosystem; biometrics; security analysis; cryptographic protocol; Petri nets.

\section{INTRODUCTION}

Due to the unique characteristics possessed by cryptographic protocols, analysis and evaluation tend to be more difficult than normal protocols. Typically cryptographic protocols, also known as security protocols, tend to inhabit a complex environment by utilising various cryptographic mechanisms, such as symmetric and asymmetric encryption, hash functions, timestamps, and digital signature [1]. For this reason, Petri nets offer the opportunity to conduct an in-depth analysis and overcome security vulnerabilities and weaknesses. Moreover, they simplify the modelling of exchange messages between nodes and describe behaviour of authentication and key agreement procedure. A number of researchers have used Petri nets to model and analyse cryptographic protocols [2 -6].

The structure of this paper is organised as follows. In Section 2, we briefly review previous works on Petri nets and our new protocol. In Section 3, we model the client-server trust model using PN. In Section 4, we add the adversary entity to the trust model and simulate various attacks using PN. We then provide a brief discussion on security analysis in Section 5. Finally, the conclusions are given in Section 6.

\section{REVIEW OF RELATED WORK}

\section{A. Petri Nets}

The concept of the Petri net [7] was introduced in 1962 by Carl Adam Petri [8]. Petri nets are graphical diagrammatic tools based on strong mathematical foundations. It is used as a visual communication aid to model concurrency, synchronisation, limited resources, sequentially, mutual exclusion and behaviour in distributed systems [9-11]. A Petri net is defined as a bipartite directed, weighted graph with two types of nodes called places and transitions, linked by directed arcs. In other words, a Petri net must consist of the following components [9-11]:

- A set of places (drawn as circles in the graphical representation), represent conditions and possible states of the system.

- A set of transitions (drawn as rectangles or thick bars), represent a change of state which caused by events or actions

- A set of arcs (drawn as arrows), connecting a place to transition and vice versa.

- Tokens (drawn as black dots), occupy places to represent the truth of the associated condition.

The formal definition of a Petri net is shown in Table 1 [10]. Generally Petri nets focus on specific properties such as liveness, deadlock, livelock, boundedness and safeness [9-11].

Table 1. Formal Definition of a Petri Net

\begin{abstract}
A Petri net structure $N=(P, T, F, W)$ without any specific initial marking is denoted by $N$.

A Petri net with the given initial marking is denoted by $\left(N, M_{0}\right)$.
\end{abstract}

Petri nets are used in this paper to ensure the soundness of the protocol analysis. This approach is a very useful tool for modelling and simulating a range of possible attacks on the proposed protocol. The key features of using Petri nets can be summarised as follows:

1. The ability to model the concurrency of the protocol progress with tokens

2. The ability to model intermediate and final objectives as places 
3. The ability to model transitions as commands and inputs

\section{B. Review of Proposed Protocl}

In our previous work [12], we have developed a new authentication protocol that allows remote mutual authentication with key agreement. Our new protocol is based on biometric verification and ID-based Cryptograph [13].
Moreover, the new protocol is aimed to initiate secure authentication and communication between the client and server by building a robust mechanism between communicating parties The proposed protocol may be described as a two-factor user authentication mechanism and three-way handshake procedure to establish a reliable connection and ensure secure data sharing. Our new protocol consists of four phases: system initialising phase, registration phase, login phase, and authentication phase. The new protocol is summarised in Fig. 1 and the notations used for the new protocol are summarised in Table 2.

\begin{tabular}{|c|c|c|c|}
\hline 节 & $\begin{array}{l}\text { Client } \mathbf{C}_{\mathbf{i}} \\
\text { (1) } \mathrm{ID}_{\mathrm{ci}}, \mathrm{PW}_{\mathrm{ci}}, \mathrm{Bio}_{\mathrm{ci}}\end{array}$ & $\begin{array}{l}\text { (3) } \operatorname{ID}_{\mathrm{C}_{\mathrm{i}},} \mathrm{H}_{4}(.), \operatorname{Enc}\{\} \mathrm{a} / \operatorname{Dec}\{\} \mathrm{a}, \\
\mathrm{f}_{\mathrm{i}}, \mathrm{e}_{\mathrm{i}}, \tau, \operatorname{Pr} \_\mathrm{K}_{\mathrm{C}_{\mathrm{i}}}\end{array}$ & $\begin{array}{l}\quad \text { Registration Centre } \mathbf{R}_{\mathbf{i}} \\
\text { (2) Computes: } \\
\text { - } \mathrm{f}_{\mathrm{i}}=\mathrm{H}_{4}\left(\mathrm{Bio}_{\mathrm{ci}}\right) \\
\quad \mathrm{e}_{\mathrm{i}}=\mathrm{H}_{4}\left(\mathrm{ID}_{\mathrm{c}}|| \mathrm{y}\right) \oplus \mathrm{H}_{4}\left(\mathrm{PW}_{\mathrm{C}}|| \mathrm{f}_{\mathrm{i}}\right) \\
\cdot \mathrm{Pr}_{-} \mathrm{K}_{\mathrm{ci}}=\left(\mathrm{x}+\mathrm{H}_{4}\left(\mathrm{ID}_{\mathrm{c}_{\mathrm{i}}}\right)\right)^{-1} . \mathrm{P}\end{array}$ \\
\hline 范 & 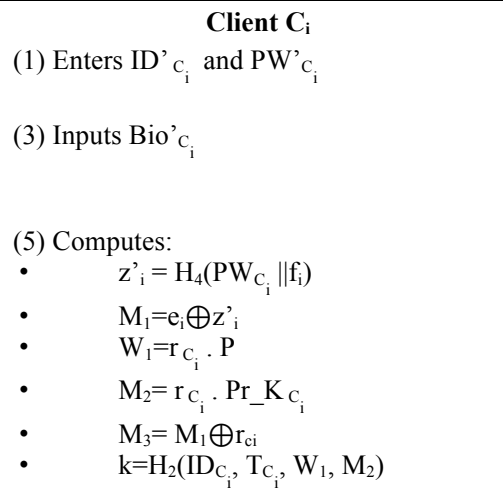 & $\begin{array}{l}(6) \mathrm{C}_{1}=\operatorname{Enc}\left\{\operatorname{ID}_{\mathrm{C}_{\mathrm{i}}}, \mathrm{T}_{\mathrm{C}_{\mathrm{i}}}, \mathrm{W}_{\mathrm{l}}, \mathrm{M}_{3},\right. \\
\underset{\left.\mathrm{MAC}_{\mathrm{k}}\left(\mathrm{ID}_{\mathrm{C}}, \mathrm{T}_{\mathrm{C}_{\mathrm{j}}}, \mathrm{W}_{1}, \mathrm{M}_{3}\right)\right\}_{\mathrm{a}}}{\longrightarrow}\end{array}$ & 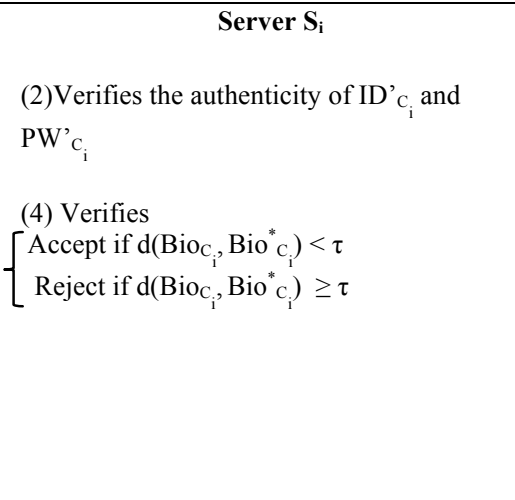 \\
\hline | & $\begin{array}{l}\text { (5) Decrypts } \mathrm{C}_{2} \text { and verifies } \mathrm{M}_{7} \text { ? }= \\
\mathrm{H}_{4}\left(\mathrm{M}_{4} \| \mathrm{r}_{\mathrm{C}_{\mathrm{i}}}\right) \text { and the integrity of } \mathrm{MAC}_{\mathrm{k}}\left(\mathrm{ID}_{\mathrm{C}_{\mathrm{i}}} \text {, }\right. \\
\left.\mathrm{T}_{\mathrm{S}_{\mathrm{i}}}, \mathrm{W}_{2}, \mathrm{M}_{6}, \mathrm{M}_{7}\right) \\
\text { Server } S_{i} \text { is authenticated } \\
\text { (6) Computes: } \\
\text { - } \quad \mathrm{K}_{\mathrm{C}_{\mathrm{i}}}=\mathrm{r}_{\mathrm{C}_{\mathrm{i}}} \cdot \mathrm{W}_{2} \\
\text { - } \quad \mathrm{Sk}=\mathrm{H}_{3}\left(\mathrm{ID}_{\mathrm{C}_{\mathrm{i}}}, \mathrm{T}_{\mathrm{C}_{\mathrm{i}}}, \mathrm{T}_{\mathrm{S}_{\mathrm{i}}}, \mathrm{W}_{1}, \mathrm{~W}_{2}, \mathrm{~K}_{\mathrm{C}_{\mathrm{i}}}\right) \\
\text { - } \quad \mathrm{M}_{8}=\mathrm{M}_{6} \oplus \mathrm{M}_{1}=\mathrm{r}_{\mathrm{S}_{\mathrm{i}}} \\
\text { - } \quad \mathrm{M}_{9}=\mathrm{H}_{4}\left(\mathrm{M}_{6} \| \mathrm{M}_{8}\right)\end{array}$ & $\begin{array}{c}\text { (4) } \mathrm{C}_{2}=\operatorname{Enc}\left\{\operatorname{ID}_{\mathrm{C}_{\mathrm{i}}}, \mathrm{T}_{\mathrm{S}_{\mathrm{i}}}, \mathrm{W}_{2}, \mathrm{M}_{6},\right. \\
\mathrm{M}_{7}, \mathrm{MAC}_{\mathrm{k}}\left(\mathrm{ID}_{\mathrm{C}_{\mathrm{i}}}, \mathrm{T}_{\mathrm{S}_{\mathrm{i}}}, \mathrm{W}_{2}, \mathrm{M}_{6},\right. \\
\left.\left.\mathrm{M}_{7}\right)\right\}_{\mathrm{a}}\end{array}$ & $\begin{array}{l}\text { Server } \mathrm{S}_{\mathrm{i}} \\
\text { (1) Decrypts } \mathrm{C}_{1}, \text { then checks validity of } \mathrm{ID}_{\mathrm{c}_{\mathrm{i}}} \\
\text { and freshness of } \mathrm{T}_{\mathrm{c}_{\mathrm{i}}} \\
\text { (2) Computes: } \\
\text { - } \mathrm{M}_{2}=\left(\mathrm{x}+\mathrm{H}_{1}\left(\mathrm{ID}_{\mathrm{C}_{\mathrm{i}}}\right)^{-1} \cdot \mathrm{W}_{1}\right. \\
\text { - } \mathrm{k}=\mathrm{H}_{2}\left(\mathrm{ID}_{\mathrm{C}_{\mathrm{i}}}, \mathrm{T}_{\mathrm{C}_{\mathrm{i}}}, \mathrm{W}_{1}, \mathrm{M}_{2}\right) \\
\text { - Checks the integrity of } \mathrm{MAC}_{\mathrm{k}}\left(\mathrm{ID}_{\mathrm{C}_{\mathrm{i}}} \text {, }\right. \\
\left.\qquad \mathrm{T}_{\mathrm{C}_{\mathrm{i}}}, \mathrm{W}_{1}, \mathrm{M}_{3}\right) \\
\text { (3) Computes: } \\
\text { - } \mathrm{M}_{4}=\mathrm{H}_{4}\left(\mathrm{ID}_{\mathrm{C}_{\mathrm{i}}} \| \mathrm{y}\right) \\
\text { - } \mathrm{W}_{2}=\mathrm{r}_{\mathrm{S}_{\mathrm{i}}} \cdot \mathrm{P} \\
\text { - } \mathrm{K}_{\mathrm{S}_{\mathrm{i}}}=\mathrm{r}_{\mathrm{S}_{\mathrm{i}}} \cdot \mathrm{W}_{1} \\
\text { - } \mathrm{Sk}_{\mathrm{k}}=\mathrm{H}_{3}\left(\mathrm{ID}_{\mathrm{C}_{\mathrm{i}}}, \mathrm{T}_{\mathrm{C}_{\mathrm{i}}}, \mathrm{T}_{\mathrm{S}_{\mathrm{i}}} \mathrm{W}_{1}, \mathrm{~W}_{2}, \mathrm{~K}_{\mathrm{S}_{\mathrm{i}}}\right) \\
\text { - } \mathrm{M}_{5}=\mathrm{M}_{3} \oplus \mathrm{M}_{4}=\mathrm{r}_{\mathrm{C}_{\mathrm{i}}} \\
\text { - } \mathrm{M}_{6}=\mathrm{M}_{4} \oplus \mathrm{r}_{\mathrm{S}_{\mathrm{i}}} \\
\text { - } \mathrm{M}_{7}=\mathrm{H}_{4}\left(\mathrm{M}_{3} \| \mathrm{M}_{5}\right)\end{array}$ \\
\hline
\end{tabular}


TABLE 2. NOTATIONS USED IN THE NEW PROTOCOL

\begin{tabular}{cl}
\hline Symbol & Definition \\
\hline $\mathrm{C}_{\mathrm{i}}$ & User/Client /Computer \\
$\mathrm{S}_{\mathrm{i}}$ & Server \\
$\mathrm{R}_{\mathrm{i}}$ & Registration Centre \\
$\mathrm{ID}_{\mathrm{S}_{\mathrm{i}}}$ & Identity of Server \\
$\mathrm{ID}_{\mathrm{C}_{\mathrm{i}}}$ & Identity of user C \\
$\mathrm{PW}_{\mathrm{C}_{\mathrm{i}}}$ & User's password \\
Bio $_{\mathrm{C}_{\mathrm{i}}}$ & Biometric template of C \\
$\mathrm{Pub}_{-} \mathrm{K}$ & Public Key \\
$\mathrm{Pr}_{-} \mathrm{K}$ & Private Key \\
$\|$ & Message concatenation operation \\
$\mathrm{P}$ & A point on elliptic curve E with order $\mathrm{n}$ \\
$\mathrm{xP}$ & Denotes point multiplication on elliptic curve \\
$\mathrm{y}$ & A piece of secret information maintained by the server \\
$\left(\mathrm{x}, \mathrm{Pub}_{-} \mathrm{K}_{\mathrm{s}}\right)$ & The server S's Private/Public key pair, where \\
$\mathrm{r}_{\mathrm{C}_{\mathrm{i}}}, \mathrm{r}_{\mathrm{S}_{\mathrm{i}}}$ & Pub_K $\mathrm{K}_{\mathrm{s}}=\mathrm{xP}$ \\
$\mathrm{H}()$. & A random number chosen by the $\mathrm{C}_{\mathrm{i}}$ and $\mathrm{S}_{\mathrm{i}}$ respectively \\
$\mathrm{MAC} \mathrm{C}_{\mathrm{k}}(\mathrm{m})$ & A secure one-way hash function \\
$\oplus$ & The secure message authentication code of $\mathrm{m}$ under the \\
$\oplus$ & XOR operation \\
\hline
\end{tabular}

We have examined and validated the behaviour of the proposed protocol by using finite-state machines and Petri nets [14]. The following steps explain the methodology to model the proposed protocol with Petri nets:

1) Build a PN trust model of the trust relationship using TAPAAL [15] simulation and verification software. The following steps are necessary for the process of modelling:

(a) Define the places and transitions and declare their functionalities

(b) Implement a token passing scheme once the initial marking is set

(c) Assess the model's behaviour by examining reachability, boundedness, and liveness

(d) Validate the model using simulation

2) Add the adversary model. This step involves the following:

(a) Extend the original model and define places and transitions for the adversary entities

(b) Implement the token-passing scheme with the adversary

(c) Model different attack and identify any insecure behaviour

\section{Client-SERVER TRUST MOdELlED VIA PN}

The trust model is a notation for determining whom the organisations should trust with its assets. For example, organisations usually verify the applicants' resumes and references, and conduct background and history checks before trusting their employees. Once they are employed, they will be issued photo ID badges and parking permits. In contrast to the real world, it is challenging in the virtual world to identify individuals who are trusted and those who are not. A trust relationship between a client and a server can be obtained in different practices. Some systems use the traditional way that relies on passwords and digital certificates. Sometimes it may involve a trusted third party to operate the authentication and validation, such as the Kerberos login protocol [1], while other systems deploy biometric automated verification systems to recognise trusted users.

In the proposed trust model, the client-server trust relationship is initiated during the registration phase. First, the client submits his/her ID, password $\left(\mathrm{PW}_{\mathrm{C}_{\mathrm{i}}}\right)$, and biometric data $\left(\mathrm{Bio}_{\mathrm{C}_{\mathrm{i}}}\right)$. Then the server will issue in return a corresponding private key $\left(\operatorname{Pr} \mathrm{K}_{\mathrm{C}_{\mathrm{i}}}\right)$, secret key $(a)$ for the symmetric encryption, and $\tau$ predetermined threshold for biometric verification. The assumption for this model is that the client and server are trustable entities, and they never cheat. Timedarc Petri Nets are used to model the new protocol. The trust model consists of two Petri net entities: one for the client $\boldsymbol{C}$ and the other for the server $\boldsymbol{S}$. The protocol entities are derived from the protocol description in [12]. The assumption made for this model is that each legitimate participant is honest, i.e. behaves according to the protocol rules. The Petri net model in Fig. 2 represents the trust model for the proposed protocol. The definitions of the places and transitions used in this model are illustrated in Table 3 and Table 4, respectively.

Table 3. DEFINITIONS OF PLACES FOR THE TRUST MODEL

\begin{tabular}{llll}
\hline Place & Definition & Place & Definition \\
\hline$P_{1}$ & Client random number & $P_{14}$ & Encrypted SYN/ACK \\
$P_{2}$ & Client timestamp & $P_{15}$ & Decrypted SYN/ACK \\
$P_{3}$ & SYN request & $P_{16}$ & Verification message \\
$P_{4}$ & Login request & $P_{17}$ & Rejected request \\
$P_{5}$ & Encrypted login & $P_{18}$ & Accept request - Server \\
& request & & is authenticated \\
$P_{6}$ & Decrypted login req. & $P_{19}$ & Session key \\
$P_{7}$ & Verification message & $P_{20}$ & ACK \\
$P_{8}$ & Rejected request & $P_{21}$ & Encrypted ACK \\
$P_{9}$ & Accepted request & $P_{22}$ & Decrypted ACK \\
$P_{10}$ & Server random number & $P_{23}$ & Verification message \\
$P_{11}$ & Server timestamp & $P_{24}$ & Rejected request \\
$P_{12}$ & Session Key & $P_{25}$ & Accept request - Client \\
$P_{13}$ & SYN/ACK & & is authenticated \\
\hline
\end{tabular}

Table 4. DEFINITIONS OF TRANSITIONS FOR TRUST MODEL

\begin{tabular}{llcl}
\hline Trans. & Definition & Trans. & Definition \\
\hline$T_{1}$ & Compute login request + & $T_{10}$ & Split the packet and \\
& SYN & & verify \\
$T_{2}$ & Encrypt & $T_{11}$ & Drop the packet \\
$T_{3}$ & Decrypt & $T_{12}$ & Accept \\
$T_{4}$ & Split the packet and verify & $T_{13}$ & Compute ACK and \\
& & & session key \\
$T_{5}$ & Drop the request & $T_{14}$ & Encrypt ACK \\
$T_{6}$ & Accept & $T_{15}$ & Decrypt ACK \\
$T_{7}$ & Compute SYN/ACK and & $T_{16}$ & Split the packet and \\
& session key & & verify \\
$T_{8}$ & Encrypt SYN/ACK & $T_{17}$ & Drop the packet \\
$T_{9}$ & Decrypt SYN/ACK & $T_{18}$ & Accept \\
\hline
\end{tabular}




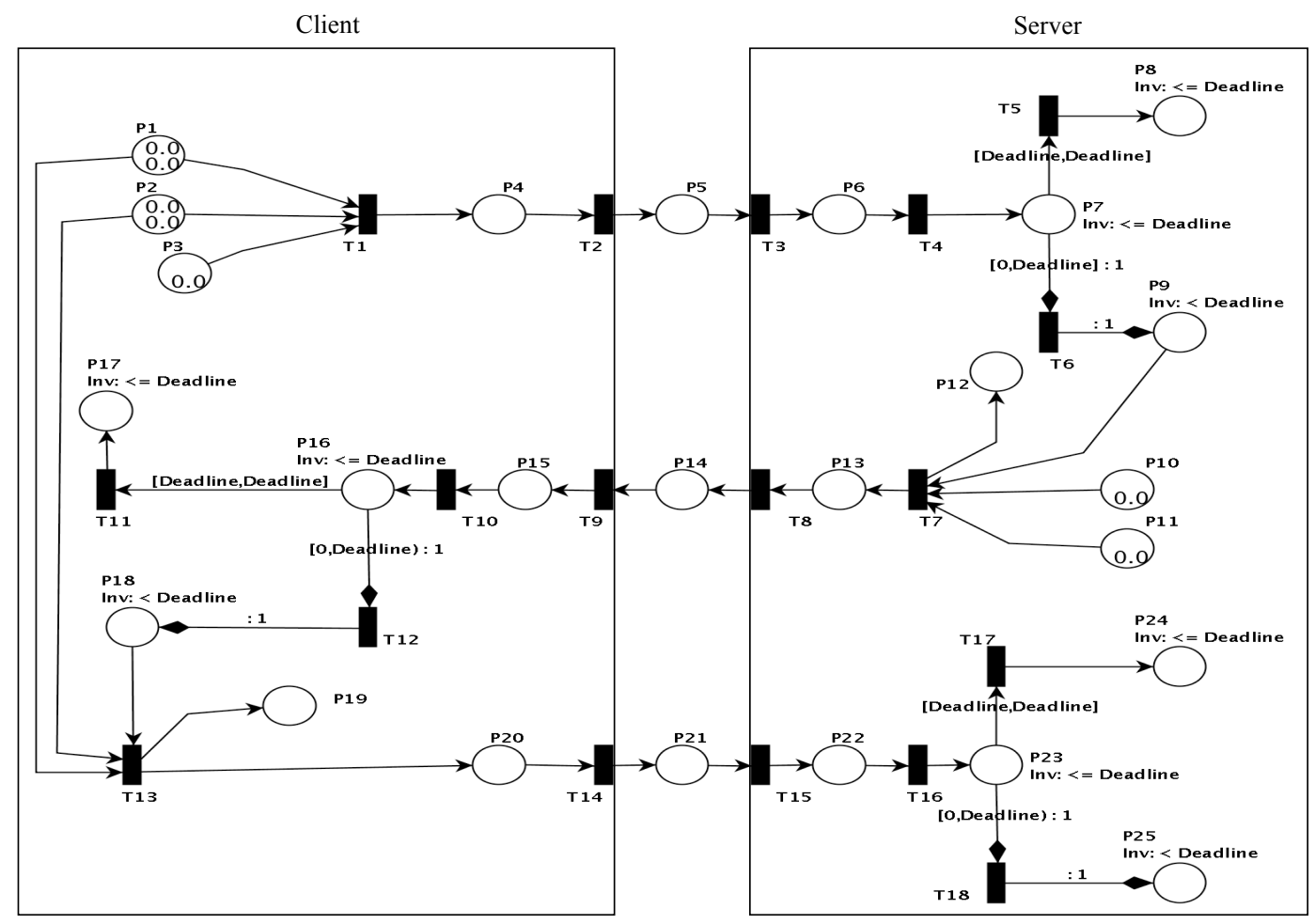

Figure 2. The client-server trust model

In the trust model, the channels between $\boldsymbol{C}$ and $\boldsymbol{S}$ are depicted by interconnected arcs, which are attached to places. The exchange messages procedure is represented by tokens. Places represent storage for requests, messages, ciphers, or session keys. Transitions in the model describe particular functions or procedures, which may be performed while in an execution state. For example, the following events produce a new state: encryption, decryption, verification, and computations. Tokens are modelled in PN as shown in Fig. 2 to represent the key agreement and message exchange between the client and server. During simulation, the token firing rule imitates the three-way handshake procedure. The structure of a place linked to a transition represents a segment of serial processes performed by the entity to fulfil its role in the protocol run. For instance, the transition $T_{1}$ in Fig. 2 consumes three tokens from $P_{1}, P_{2}$, and $P_{3}$ to calculate the login request. The PN trust model represents a three-way handshake producer between $\boldsymbol{C}$ and $\boldsymbol{S}$. It allows both $\boldsymbol{C}$ and $\boldsymbol{S}$ to agree on a shared session key over an insecure channel. The steps of protocol analysis for PN trust model are described as follows:

- At first, the protocol is initiated by a client. The client entity of the PN trust model generates a random value $\left(P_{1}\right)$, Timestamp $\left(P_{2}\right)$, SYN request $\left(P_{3}\right)$ to compute the login request $\left(P_{4}\right)$ within a certain period of time. $C$ sends the encrypted request $\left(P_{5}\right)$ to $S$.
- Upon receiving the request, $\boldsymbol{S}$ will check the age of the token. Note that, computing and sending the request to $\boldsymbol{S}$ takes some units of time. $\boldsymbol{S}$ will drop the request if the time processing exceeds the deadline. This is guaranteed by the use of transport arcs that preserve the age of the tokens and the corresponding invariants.

- In the second message of the handshake, the server entity generates a random value $\left(P_{10}\right)$, timestamp $\left(P_{11}\right)$ to compute the session key $\left(P_{12}\right)$, and SYN/ACK request $\left(P_{13}\right)$. Then $\mathrm{S}$ sends the encrypted SYN/ACK $\left(\mathrm{P}_{14}\right)$ to $\boldsymbol{C}$.

- $\quad$ Upon receiving SYN/ACK, $\boldsymbol{C}$ checks the token age and computes the session key $\left(P_{19}\right)$. At this stage, $\boldsymbol{C}$ authenticates $\boldsymbol{S}$ and sends an enciphered ACK $\left(P_{2 l}\right)$ to $\boldsymbol{S}$.

- Finally, the server entity checks the token age and authenticates $\boldsymbol{C}$.

\section{TRust Model With AdVERSARY Modelled VIA PN}

The purpose of this analysis is to find weaknesses and flaws in the proposed protocol. It is essential to examine the behaviour of the protocol with the presence of a malicious adversary. An adversary entity can be a hacker, a malicious insider, a disgruntled employee, a terrorist, organised crime, or competitors. 


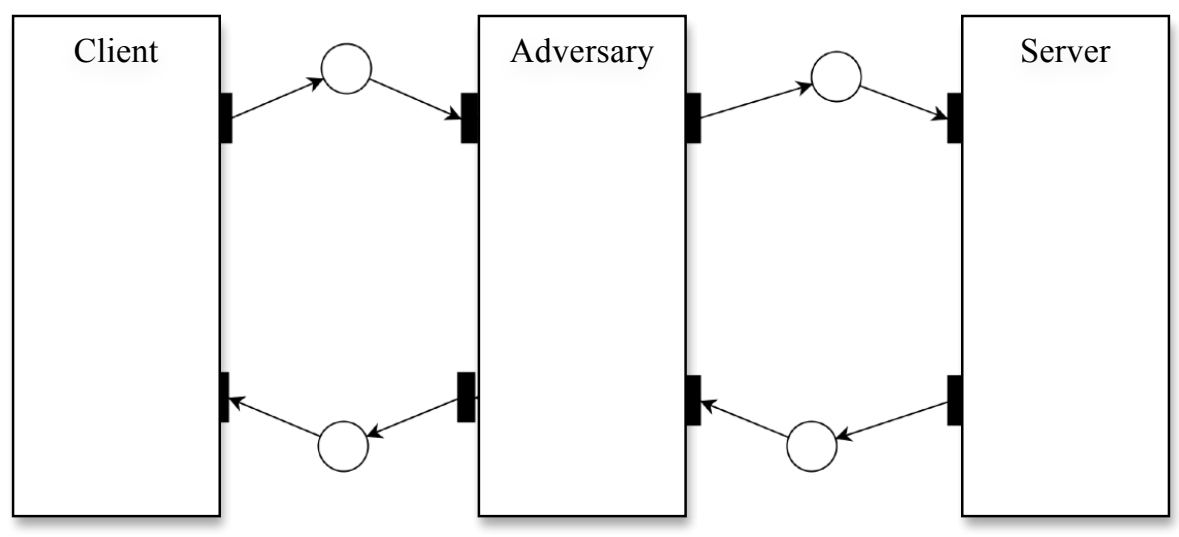

Figure 3. High-level view of adversary entity attacking the protocol

The worst-case scenario would be if attackers obtained illegitimate access to the target system. They could install malicious software, like a rootkit, to remove or modify data. This act of unauthorised access could lead to privilege escalation and allow the attacker to gain elevated entry to resources that are meant to be protected from other application users. Moreover, faulty protocols may allow an attacker to compromise other machines in the network to act as zombie computers to launch denial-of-service attacks.

PN modelling is capable of mapping out how messages flow throughout the protocol with an adversary. A high-level view of the adversary model with information flow is shown in Fig. 3.

The adversary entity is composed of processes, each designed for a specific function in the protocol. Each process models the adversary's possible actions to capture tokens. It can intercept messages from the channel, alter them, and pass them to the target source.

Conceptually, the adversary entity is nondeterministic, in that it may perform different possible actions under different client identities at a given time to ultimately compromise the target system. The following assumptions are considered for the adversary model:

1) The adversary can eavesdrop, intercept, and store messages. It may block or pass any of these messages. Additionally, it may construct forged messages from captured data and inject them into the channel.

2) The adversary has zero knowledge such that it does not possess any elements of messages transmitted between the legitimate nodes but it can learn by observing the traffic.

3) The traffic between client and server is not encrypted.

The main goal of the adversary model is to examine the protocol behaviour with the presence of an adversary while modelling attacks. In the adversary model (attack model), the description of client and server entities is similar to the trust model descried in section 3. For adversary entity, places represent an adversary database, which store, control, knowledge and accumulate all the intercepted messages.
Transitions represent a set of input events and commands the adversary may perform to launch an attack. The input token in the adversary entity indicates that the message has been captured. The token movement from place to place through the directed arcs indicates the progress of an attack. To distinguish a genuine traffic from forged traffic, the grave symbol ' is used to indicate that the variable could be modified. For example, if the adversary intercepts the message $[\mathrm{A}, \mathrm{B}, \mathrm{C}]$, the output message would be [A', B', C'], which means the message has been manipulated by the adversary.

\section{A. Analysis of Man-in-the-Middle Attack}

After adding an adversary entity to the model, it can be noticed that there is the possibility of a man-in-the-middle between the two entities $\boldsymbol{C}$ and $\boldsymbol{S}$. An active adversary $\boldsymbol{A}$ can intercept the communication line between a legitimate client and a trusted server as well as manipulate the protocol by using some means to successfully masquerade either as server or client. The attack model in Fig. 4 represents the man-in-themiddle attack for the proposed protocol. The definitions of the places and transitions used in this model are illustrated in Table 5 and Table 6 , respectively.

Table 5. DEFINITIONS OF TRANSITIONS - MAN-IN-THEMIDDLE ATTACK MODEL

\begin{tabular}{llll}
\hline Trans. & \multicolumn{1}{c}{ Definition } & Trans. & Definition \\
\hline$T_{1}$ & Compute login request & $T_{13}$ & Send forge SYN/ACK \\
& + SYN & $T_{14}$ & Receive forge SYN/ACK \\
$T_{2}$ & Send MSG & $T_{15}$ & Split the packet and verify \\
$T_{3}$ & Intercept MSG & $T_{16}$ & Drop the request \\
$T_{4}$ & Duplicate MSG & $T_{17}$ & Accept \\
$T_{5}$ & Send forge MSG & $T_{18}$ & Compute ACK and \\
$T_{6}$ & Received Forge MSG & & session key \\
$T_{7}$ & Split the packet and & $T_{19}$ & Send ACK \\
& verify & $T_{20}$ & Intercept MSG \\
$T_{8}$ & Drop the request & $T_{21}$ & Send forge ACK \\
$T_{9}$ & Accept & $T_{22}$ & Receive forge ACK \\
$T_{10}$ & Compute SYN/ACK & $T_{23}$ & Split the packet and verify \\
& and session key & $T_{24}$ & Drop the request \\
$T_{11}$ & Send SYN/ACK & $T_{25}$ & Accept \\
$T_{12}$ & Intercept MSG & & \\
\hline
\end{tabular}


Table 6. DEFINITIONS OF PLACES - THE MAN-IN-THEMIDDLLE MODEL

\begin{tabular}{llcl}
\hline Place & \multicolumn{1}{c}{ Definition } & Place & Definition \\
\hline$P_{1}$ & Client random number & $P_{22}$ & Sent SYN/ACK for A \\
$P_{2}$ & Client timestamp & $P_{23}$ & Sent SYN/ACK for C \\
$P_{3}$ & SYN request & $P_{24}$ & Received SYN/ACK for C \\
$P_{4}$ & Login request & $P_{25}$ & Received SYN/ACK for A \\
$P_{5}$ & Sent request & $P_{26}$ & Sent forge SYN/ACK to C \\
$P_{6}$ & Intercepted MSG & $P_{27}$ & Received forge SYN/ACK \\
$P_{7}$ & Forge MSG A & $P_{28}$ & Verification message \\
$P_{8}$ & Forge MSG C & $P_{29}$ & Rejected request \\
$P_{9}$ & Sent forge MSG A & $P_{30}$ & Accept request - A is \\
$P_{10}$ & Sent forge MSG C & & authenticated \\
$P_{11}$ & Received forge MSG A & $P_{31}$ & Session key \\
$P_{12}$ & Received forge MSG A & $P_{32}$ & ACK \\
$P_{13}$ & Verification message & $P_{33}$ & Sent ACK \\
$P_{14}$ & Rejected request & $P_{34}$ & Intercepted ACK \\
$P_{15}$ & Accepted request & $P_{35}$ & Forge ACK \\
$P_{16}$ & Server random number & $P_{36}$ & Received forge ACK \\
$P_{17}$ & Server timestamp & $P_{37}$ & Verification message \\
$P_{18}$ & A Session Key & $P_{38}$ & Rejected request \\
$P_{19}$ & C Session key & $P_{39}$ & Accept request - A is \\
$P_{20}$ & SYN/ACK for A & & authenticated \\
$P_{21}$ & SYN/ACK for C & & \\
\hline
\end{tabular}

According to Fig. 4 the man-in-the-middle attack proceeds as follows:

- In the login phase, when the client $\boldsymbol{C}$ initiates and sends the login request $\left(P_{4}\right)$ to the server $\boldsymbol{S}$, an adversary $\boldsymbol{A}$ may intercept the login message. Transition $\mathrm{T}_{3}$ represents the initial phase of the attack. $\boldsymbol{A}$ can duplicate the login message and then start two sessions with $S$ by sending two copies of request: $P_{7}=P_{8}=\left[\mathrm{ID}_{\mathrm{C}}{ }_{\mathrm{C}}, \mathrm{T}_{\mathrm{C}}{ }_{\mathrm{C}}, \mathrm{W}^{\prime}{ }_{1}, \mathrm{M}_{3}{ }_{3}\right.$, $\left.\mathrm{MAC}_{\mathrm{k}}{ }_{\mathrm{k}}\left(\mathrm{ID}_{\mathrm{C}}, \mathrm{T}_{\mathrm{C}}, \mathrm{W}_{1}, \mathrm{M}_{3}\right)\right]$ to $\boldsymbol{S}$.

- $\quad$ Upon receiving $\left(P_{11}\right)$ and $\left(P_{12}\right), \boldsymbol{S}$ generates two random numbers and two timestamps and computes the following:

○ Two session keys $\left(P_{18}, P_{19}\right)$ for $\boldsymbol{A}$ and $\boldsymbol{C}$, respectively

- Two SYN/ACK messages $\left(P_{20}, P_{2 l}\right)$ for $\boldsymbol{A}$ and $C$, respectively

Then, $\boldsymbol{S}$ sends the messages $\left(P_{22}, P_{23}\right)$ for the two sessions respectively.

- In the meantime, $\boldsymbol{A}$ captures $\left(P_{22}, P_{23}\right)$ and sends a forged message $\left(P_{25}\right)$ to $\boldsymbol{C}$.

- $\quad$ After receiving the $\left(P_{27}\right), \boldsymbol{C}$ verifies it, which in this case is a genuine request $\left[\mathrm{ID}_{\mathrm{C}}, \mathrm{T}_{\mathrm{S}}, \mathrm{W}_{2}, \mathrm{M}_{6}, \mathrm{M}_{7}, \mathrm{MAC}_{\mathrm{k}}\left(\mathrm{ID}_{\mathrm{C}}, \mathrm{T}_{\mathrm{S}}\right.\right.$, $\left.\left.\mathrm{W}_{2}, \quad \mathrm{M}_{6}, \mathrm{M}_{7}\right)\right]$. Consequently, $\boldsymbol{C}$ authenticates $\boldsymbol{A}$ masquerading as $\boldsymbol{S}$. Then $\boldsymbol{C}$ computes the shared session key $\left(P_{31}\right)$ and sends ACK $\left(P_{32}\right)$ to $\boldsymbol{S}$.

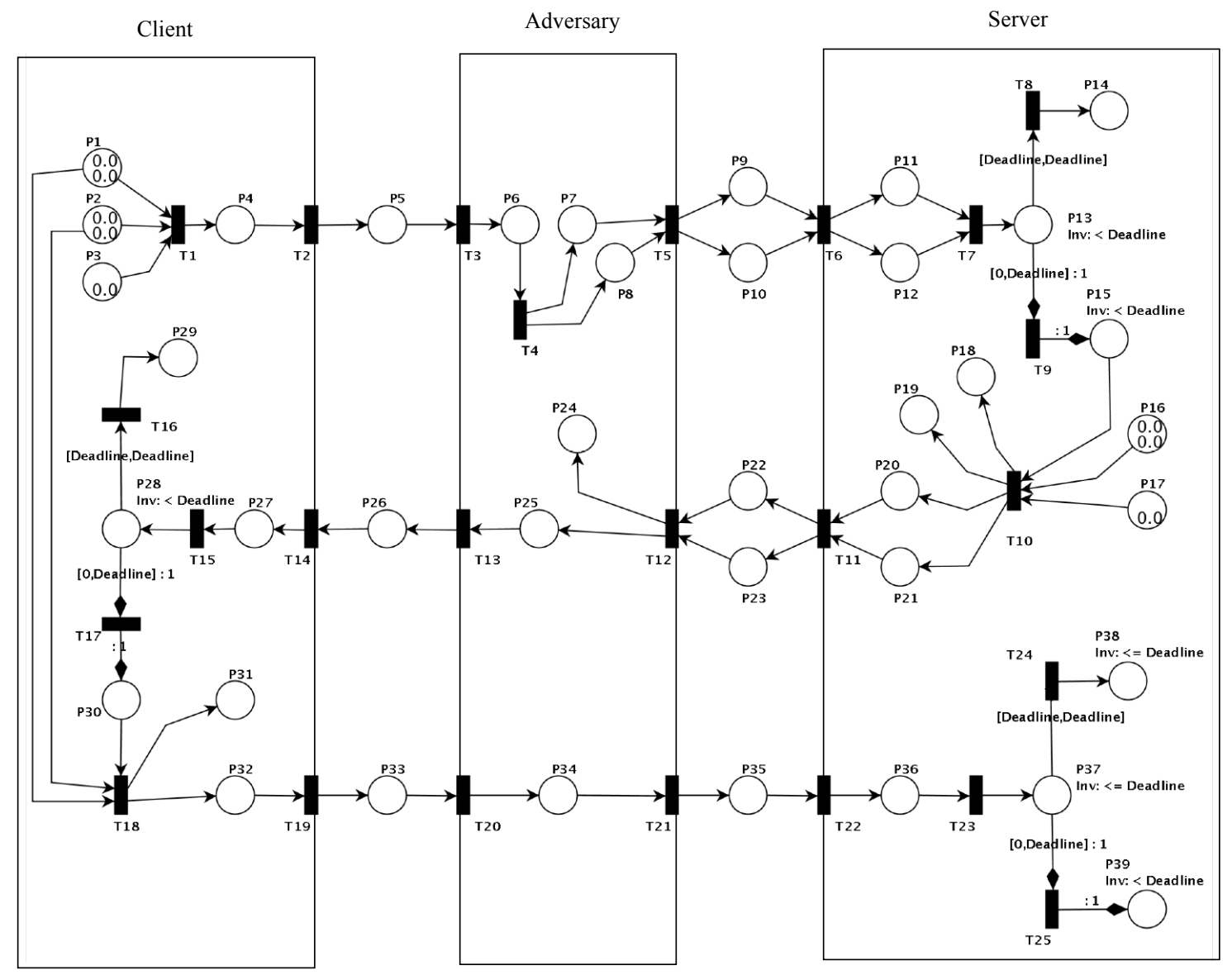

Figure 4. Modelling man-in-the-middle attack 
- $\quad \boldsymbol{A}$ intercepts $\left(P_{32}\right)$ and forwards it to $\boldsymbol{S}$.

- After receiving $\left(P_{36}\right), \boldsymbol{S}$ verifies $\mathrm{ACK}=\mathrm{H}_{4}\left(\mathrm{M}_{6} \oplus \mathrm{r}_{\mathrm{S}}\right)$. Thus, $\boldsymbol{A}$ is successfully authenticated by $\boldsymbol{S}$ masquerading $\boldsymbol{C}$.

By analysing the protocol, without encrypting the traffic, the proposed protocol is prone to man-in-the-middle attack. The adversary has the ability to control the negotiation between the client and the server. In fact, the adversary can clearly modify, substitute or delete all subsequent messages. It is obvious that both the client and the server have established a bogus session with the adversary.

\section{B. Analysis of Reflection Attack}

The reflection attack consists of two parties. The adversary in this model is masquerading as the server. In this PN model, places represent either input or output of protocol run. Transitions are used to explicit the client and adversary actions. Tokens indicate the progress of the attack. Fig. 4 describes the execution of a reflection attack for the proposed protocol with presence of the client and adversary. The definitions of the places and the transitions used in this model are illustrated in Table 7 and Table 8, respectively.
Table 7. DEFINITIONS OF PLACES - THE REFLECTION ATTACK MODEL

\begin{tabular}{llcl}
\hline Place & \multicolumn{1}{c}{ Definition } & Place & Definition \\
\hline$P_{1}$ & Client random number & $P_{9}$ & Received forge SYN/ACK \\
$P_{2}$ & Client timestamp & $P_{10}$ & Verification message \\
$P_{3}$ & SYN request & $P_{11}$ & Rejected request \\
$P_{4}$ & Login request & $P_{12}$ & Accepted request \\
$P_{5}$ & Sent request & $P_{13}$ & Session key \\
$P_{6}$ & Intercepted MSG & $P_{14}$ & ACK \\
$P_{7}$ & Sent forge SYN/ACK & $P_{15}$ & Sent ACK \\
$P_{8}$ & Received forge & $P_{16}$ & Received ACK \\
& SYN/ACK & & \\
\hline
\end{tabular}

Table 8. DEFINITIONS OF TRANSITIONS - REFLECTION ATTACK MODEL

\begin{tabular}{llcl}
\hline Trans. & \multicolumn{1}{c}{ Definition } & Trans. & Definition \\
\hline$T_{1}$ & Compute login request & $T_{7}$ & Split the packet and verify \\
& and SYN & $T_{8}$ & Drop the request \\
$T_{2}$ & Send MSG & $T_{9}$ & Accept the request \\
$T_{3}$ & Intercept MSG & $T_{10}$ & Compute ACK and \\
$T_{4}$ & Fabricate SYN/ACK & & session key \\
$T_{5}$ & Send fake SYN/ACK & $T_{11}$ & Send ACK \\
$T_{6}$ & Received fake & $T_{12}$ & Receive ACK \\
& SYN/ACK & & \\
\hline
\end{tabular}

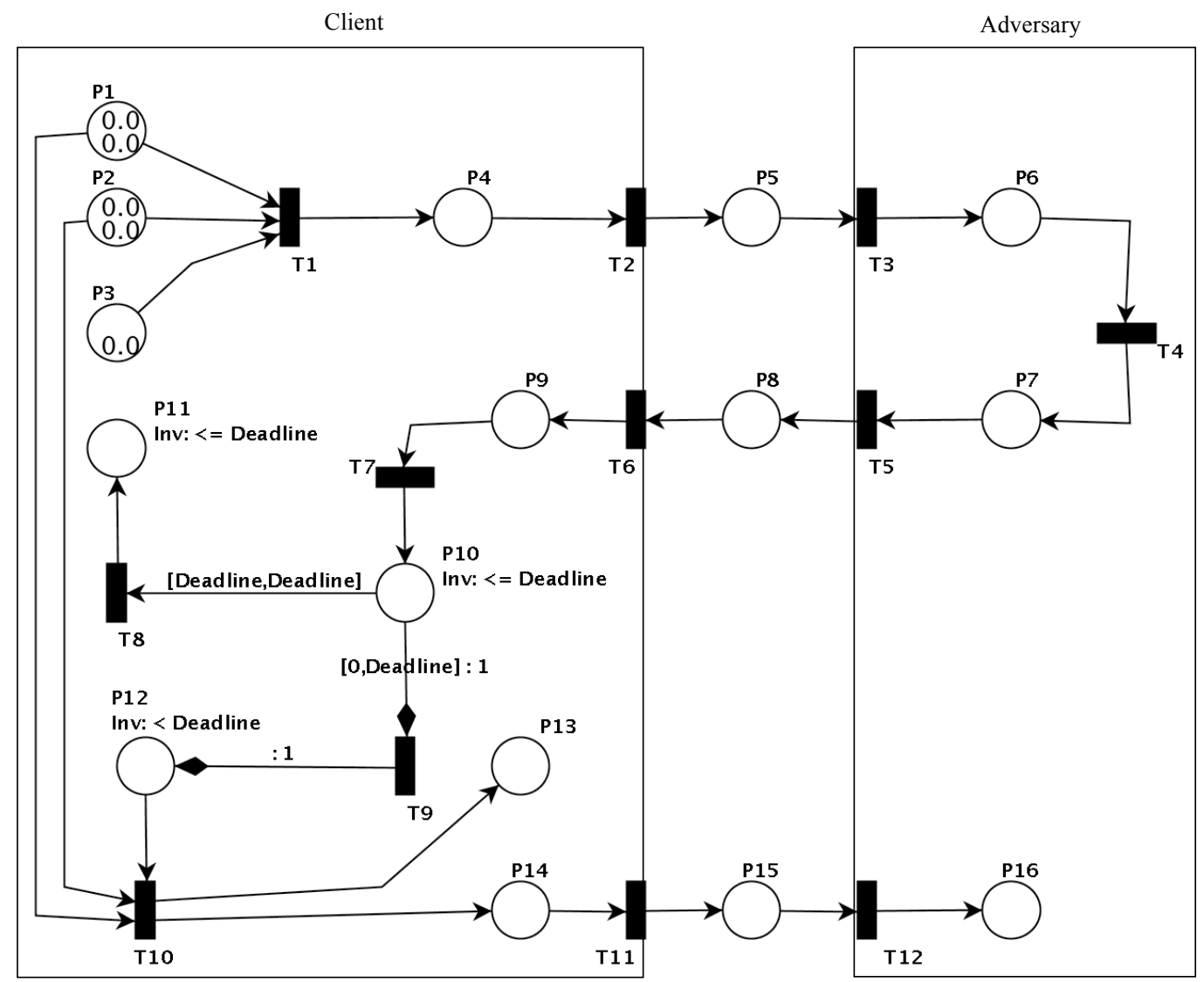

Figure 5. Modelling reflection attack 
Since client $C$ 's login request $\left[\mathrm{ID}_{\mathrm{C}}, \mathrm{T}_{\mathrm{C}}, \mathrm{W}_{1}, \mathrm{M}_{3}\right.$, $\left.\mathrm{MAC}_{\mathrm{k}}\left(\mathrm{ID}_{\mathrm{C}}, \mathrm{T}_{\mathrm{C}}, \mathrm{W}_{1}, \mathrm{M}_{3}\right)\right]$ is symmetrical to server $\boldsymbol{S}$ response $\left[\mathrm{ID}_{\mathrm{C}}, \mathrm{T}_{\mathrm{S}}, \mathrm{W}_{2}, \mathrm{M}_{6}, \mathrm{M}_{7}, \mathrm{MAC}_{\mathrm{k}}\left(\mathrm{ID}_{\mathrm{C}}, \mathrm{T}_{\mathrm{S}}, \mathrm{W}_{2}, \mathrm{M}_{6}, \mathrm{M}_{7}\right)\right]$ but the differences between them can be only found in the timestamps and hash values. This symmetry flaw leads to reflection attack. To exploit the reflection attack, the adversary $\boldsymbol{A}$ intercepts the login request while listening to the electronic conversation between client $\boldsymbol{C}$ and server $\boldsymbol{S}$. Then, the adversary sends the same login request $\left[\mathrm{ID}_{\mathrm{C}}{ }_{\mathrm{C}}, \mathrm{T}_{\mathrm{C}}{ }_{\mathrm{C}}, \mathrm{W}_{1}{ }_{1}, \mathrm{M}_{3}{ }_{3}, \mathrm{MAC}_{\mathrm{k}}{ }_{\mathrm{k}}\left(\mathrm{ID}_{\mathrm{C}}, \mathrm{T}_{\mathrm{C}}, \mathrm{W}_{1}\right.\right.$ , $\left.\mathrm{M}_{3}\right)$ ] to $C$ in a timely manner.

It is obvious that, upon receiving the forged server's response (which is in fact the adversary's reply request), $\boldsymbol{C}$ will automatically acknowledge the response since the computation is accomplished with the correct key, so the MAC integrity check will succeed. Consequently, $\boldsymbol{A}$ successfully masquerades as $\boldsymbol{S}$ and the protocol fails to provide mutual authentication.

Although, $\boldsymbol{A}$ can cheat $\boldsymbol{C}$ into believing it is communicating with $\boldsymbol{S}, \boldsymbol{A}$ cannot obtain the corresponding session key $s k$. Still this type of attack is deemed to represent a breach of the basic obligation of mutual authentication with limited damage. $\boldsymbol{A}$ performed the exploit without the knowledge of key $k$, merely by intercepting the challenge and sending it back to $\boldsymbol{C}$.

\section{Analysis of Parallel Session Attack}

Another attack, which is effective against the proposed model without encrypted traffic, is parallel session attack. This attack uses deception to compromise authentication protocols. It involves selecting a valid combination of information from ongoing protocol executions. Fig. 6 explains the exploitation of parallel session attack on the proposed protocol with presence of adversary. The message exchange in this attack is mainly between the server and the adversary leaving the client completely out of the picture. The definitions of the places and the transitions for this model are defined in Table 9 and Table 10 , respectively.

Table 9. DEFINITIONS OF PLACES - PARALLEL SESSION ATTACK MODEL

\begin{tabular}{llcl}
\hline Place & Definition & Place & Definition \\
\hline$P_{1}$ & Client random number & $P_{16}$ & Sent SYN/ACK \\
$P_{2}$ & Client timestamp & $P_{17}$ & Received SYN/ACK \\
$P_{3}$ & SYN request & $P_{18}$ & Fabricated Fake SYN \\
$P_{4}$ & Login request & $P_{19}$ & Sent fake SYN \\
$P_{5}$ & Sent request & $P_{20}$ & Received fake SYN \\
$P_{6}$ & Intercepted MSG & $P_{21}$ & Verification message \\
$P_{7}$ & Forge MSG & $P_{22}$ & Rejected request \\
$P_{8}$ & Sent Forge MSG & $P_{23}$ & Accepted request \\
$P_{9}$ & Verification message & $P_{24}$ & Server random number \\
$P_{10}$ & Rejected request & $P_{25}$ & Server timestamp \\
$P_{11}$ & Accepted request & $P_{26}$ & Session Key \\
$P_{12}$ & Server random number & $P_{27}$ & SYN/ACK \\
$P_{13}$ & Server timestamp & $P_{28}$ & Sent SYN/ACK \\
$P_{14}$ & Session Key & $P_{29}$ & Received SYN/ACK \\
$P_{15}$ & SYN/ACK & & \\
\hline
\end{tabular}

Table 10. DEFINITIONS OF TRANSITIONS - PARALLEL SESSION ATTACK MODEL

\begin{tabular}{llcl}
\hline Trans. & Definition & Trans. & Definition \\
\hline$T_{1}$ & Compute login request & $T_{10}$ & Send SYN/ACK \\
& + SYN & $T_{11}$ & Intercept MSG \\
$T_{2}$ & Send MSG & $T_{12}$ & Fabricate SYN \\
$T_{3}$ & Intercept MSG & $T_{13}$ & Send fake SYN \\
$T_{4}$ & Send forge MSG & $T_{14}$ & Receive forge SYN \\
$T_{5}$ & Received Forge MSG & $T_{15}$ & Split the packet and verify \\
$T_{6}$ & Split the packet and & $T_{16}$ & Drop the request \\
& verify & $T_{17}$ & Accept \\
$T_{7}$ & Drop the request & $T_{18}$ & Compute SYN/ACK and \\
$T_{8}$ & Accept & & session key \\
$T_{9}$ & Compute SYN/ACK & $T_{19}$ & Send SYN/ACK \\
& and session key & $T_{20}$ & Receive SYN/ACK \\
\hline
\end{tabular}

In the authentication phase of the proposed protocol, the adversary $\boldsymbol{A}$ can masquerade as an authorised client without prior knowledge of the password or biometric data. The exploit starts when $\boldsymbol{A}$ eavesdrops on the communication between $\boldsymbol{C}$ and $\boldsymbol{S}$. $\boldsymbol{A}$ intercepts and blocks the $\boldsymbol{S}$ response message: $P_{16}=\left[\mathrm{ID}_{\mathrm{C}_{\mathrm{i}}}, \mathrm{T}_{\mathrm{S}_{\mathrm{i}}}, \mathrm{W}_{2}, \mathrm{M}_{6}, \mathrm{M}_{7}, \mathrm{MAC}_{\mathrm{k}}\left(\mathrm{ID}_{\mathrm{C}_{\mathrm{i}}}, \mathrm{T}_{\mathrm{S}_{\mathrm{i}}}, \mathrm{W}_{2}\right.\right.$, $\left.\mathrm{M}_{6}, \mathrm{M}_{7}\right)$ ]. Then, $\boldsymbol{A}$ instantly impersonates $\boldsymbol{C}$ and initiates a new session with $\boldsymbol{S}$ by sending a fabricated login request: $P_{19}=$ $\left[\mathrm{ID}_{\mathrm{A}}=\mathrm{ID}_{\mathrm{C}}{ }_{\mathrm{C}}, \mathrm{T}_{\mathrm{A}}=\mathrm{T}_{\mathrm{s}}{ }_{\mathrm{s}}, \mathrm{W}_{1}=\mathrm{W}_{2}{ }_{2}, \mathrm{M}_{3}=\mathrm{M}_{6}{ }_{6}, \mathrm{M}_{7}, \mathrm{MAC}_{\mathrm{k}}{ }_{\mathrm{k}}\left(\mathrm{ID}_{\mathrm{C}}\right.\right.$, $\left.\mathrm{T}_{\mathrm{S}}, \mathrm{W}_{2}, \mathrm{M}_{6}, \mathrm{M}_{7}\right)$ ], which is $\boldsymbol{S}$ original reply to $\boldsymbol{C}$.

Assume if the fabricated message arrives to $S$ at time $T$, it will pass the verification check for the following reasons:

(1) The likelihood of correlation associated with $T^{\prime}-T_{C} \leq$ $\Delta T$ will be high considering the time-delay in widearea networks is unpredictable and varies most of the time. Thus, $\Delta T$ is often set higher than the timespan of a complete round-trip [16-18]

(2) The MAC integrity check will give a positive result

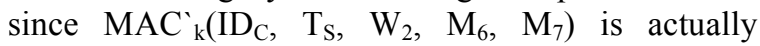
computed with the correct key $k$ by $\boldsymbol{S}$.

Based on the above assumptions, $\boldsymbol{S}$ generates random number $P_{24}$ and timestamp $P_{25}$ to computes session key $P_{26}$ and SYN/ACK response $P_{28}$, and sends it to $\boldsymbol{A}$.

\section{Analysis of Impersonation Attack}

One possible attack against the proposed model is impersonation attack. Based on the simulation of man-in-themiddle attack, reflection attack, and parallel session attack, the model reveals a potential risk and weakness that lead to impersonation attack. The adversary $\boldsymbol{A}$ can mount impersonation attack without knowing any other secret information or credentials by intercepting the login request $\left[\mathrm{ID}_{\mathrm{C}}, \mathrm{T}_{\mathrm{C}}, \mathrm{W}_{1}, \mathrm{M}_{3}, \mathrm{MAC}_{\mathrm{k}}\left(\mathrm{ID}_{\mathrm{C}}, \mathrm{T}_{\mathrm{C}}, \mathrm{W}_{1}, \mathrm{M}_{3}\right)\right]$. Hence, $\boldsymbol{A}$ can exploit the proposed protocol by using any of the methods explained previously and hijacking sessions transmitted between $\boldsymbol{C}$ and $\boldsymbol{S}$. Eventually, $\boldsymbol{A}$ succeeds to impersonating either the client or the server. 


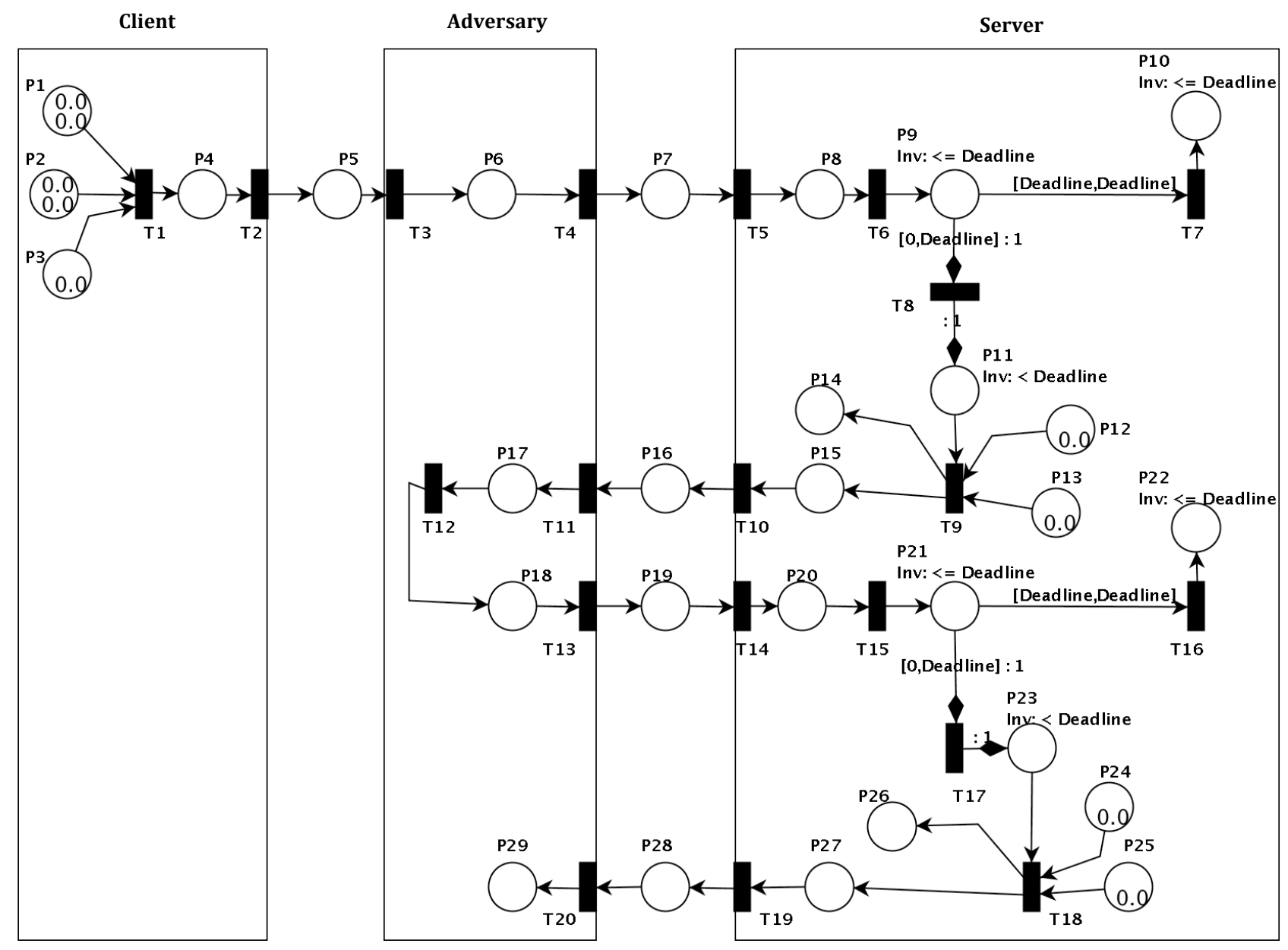

Figure 6. Modelling parallel session attack

\section{SECURITY ANALYSIS AND DISCUSSION}

Security analysis is a crucial significant process in evaluating communication and cryptographic protocols. The flaws within the protocol can be quickly removed via two solutions. First, encrypting the traffic between client and server creates a private channel to transmit a confidential conversation and calculate the session key. This mechanism is the most cost-effective solution. It is insignificant if the adversary gets hold of an encrypted form of sensitive data as long as it does not obtain the corresponding decryption key. In the second solution, the absence of server identity allows an adversary to simply masquerade as a trusted server. It is possible to optimise the protocol with a simple technique such as adding the server $\mathrm{ID}_{\mathrm{S}}$, which can fix the problem. Encrypting traffic protects client's anonymity; user anonymity is one of the security features of remote login system.

Variations of these attacks can be modelled in all phases of mutual authentication and key agreement of cycle. Modelling and simulation revealed that the unencrypted traffic does not provide a full secure authentication and permit a sensitive credential information travel in clear.
Replay Attack. The security feature in the proposed protocol can withstand replay attack due to the use of freshness property. This is guaranteed by applying timestamps and random numbers for each authentication session. To validate the authenticity of messages exchanged between $\mathbf{C}$ and $\mathbf{S}$, the freshness of timestamps is constantly checked. For example, the verification request will fail if $\mathrm{T}^{\prime}-\mathrm{T}_{\mathrm{C}}>\Delta \mathrm{T}$. This will cause the session to be terminated. Moreover, a new session key is constructed in every authentication cycle. It is worth to mentioning that, the adversary cannot compromise the old session key because it is never been transmitted in the protocol execution between the client and the server. One of the new protocol merits is that each entity computes the correct session key based on the information exchanged between them.

Forgery Attack. The adversary $\boldsymbol{A}$ cannot create a valid login from scratch without knowing the secret value and the private key of the client. Thus, the adversary cannot act as a legal client so the attack is not feasible. 


\section{CONCLUSION AND FUTURE WORK}

This paper presents a formal approach for enumerating the vulnerabilities and flaws in our protocol and determining suitable countermeasures to fix them. First, PN is used to model the client-server trust model. Then, an adversary entity is added to trust model to analyse various attacks and understand possible behaviours of the adversary. Each attack scenario has been simulated using $\mathrm{PN}$ to exploits vulnerabilities in case if the symmetric encryption was not applied to our new protocol.

Adding an adversary to the model encourages discovering and discussing scenarios where the system was under malicious attack. The range of attacks tested the behaviour of the protocol and helped to understand possible behaviours of the adversary during attacks. Each attack scenario has been simulated using PN to exploits vulnerabilities in case if the symmetric encryption was not applied to our new protocol.

It is evident that the most viable countermeasure to defend authentication attacks is to encrypt the message exchange between the client and server. Since the traffic is encrypted between the client and server, this proves that our new protocol is resistant to man-in-the-middle attack, reflection attack, parallel session attack, and impersonation attack. Also, this paper shows that replay attack and forgery attack are not effective because of the freshness property and the difficulty of creating a login request without learning any prior credentials. This analysis shows that our protocol is efficient and provides secure communication over insecure channels.

Future work will include examining ciphertext attack where the adversary can eavesdrop and intercept encrypted messages. PN will be used for modelling and simulating the attack. Once the security analysis is completed, any modification will be considered to enhance our protocol, such as including server ID in the protocol. Consequently a complex client-server trust model will be simulated and validated via $\mathrm{PN}$.

\section{ACKNOWLEDGMENT}

This research has been funded by Saudi Arabian Cultural Bureau in London and King Abdul Aziz University in Saudi Arabia.

\section{REFERENCES}

[1] Ryan, P. and Schneider, S.A., 2001. The modelling and analysis of security protocols: the csp approach. Addison-Wesley Professional

[2] Nieh, B.B. and Tavares, S.E., 1993. Modelling and analyzing cryptographic protocols using Petri Nets, Advances in CryptologyAUSCRYPT'92 1993, Springer, pp. 275-295.

[3] Al-Azzoni, I., Down, D.G. and Khedri, R., 2005. Modeling and verification of cryptographic protocols using coloured petri nets and design/CPN. Nordic Journal of Computing, 12(3), pp. 201.

[4] Dresp, W., 2005. Security analysis of the secure authentication protocol by means of coloured petri nets, Communications and Multimedia Security 2005, Springer, pp. 230-239.

[5] Permpoontanalarp, Y. and Sornkhom, P., 2009. A new Coloured Petri net methodology for the security analysis of cryptographic protocols, Proceedings of the 10th Workshop and Tutorial on Practical Use of Coloured Petri Nets and the CPN Tools, Aarhus, Denmark 2009.

[6] Xu, Y. and Xie, X., 2011. Modeling and analysis of security protocols using colored petri nets. Journal of Computers, 6(1), pp. 19-27

[7] Petri, C.A., 1962. Kommunikation mit Automaten. Ph. D. Thesis, University of Bonn.

[8] Silva, Manuel. "50 years after the PhD thesis of Carl Adam Petri: A perspective." In Discrete Event Systems, vol. 11, no. 1, pp. 13-20. 2012

[9] Peterson, J.L., 1981. Petri Net Theory and the Modeling of Systems. Prentice-Hall.

[10] Murata, T., 1989. Petri nets: properties, analysis and applications. Proceedings

[11] Bobbio, A., 1990. System modelling with Petri nets. Systems reliability assessment. Springer, pp. 103-143.

[12] Aljeaid, D., Ma, X. and Langensiepen, C., 2014. Biometric identitybased cryptography for e-Government environment, Science and Information Conference (SAI), 2014 2014, IEEE, pp. 581-588.

[13] Shamir, A., 1985. Identity-based cryptosystems and signature schemes, Advances in cryptology 1985, Springer, pp. 47-53.

[14] Aljeaid, D., Ma, X. and Langensiepen, C., Modelling and Simulation of a Biometric Identity-Based Cryptography. International Journal of Advanced Research in Artificial Intelligence (IJARAI), 3(10),.

[15] TAPAAL 2.4.3 Petri nets simulation and verfication of timed-arc Petri nets. Available ar: www.tapaal.net.

[16] Mills, D.L., 1991. Internet time synchronization: the network time protocol. Communications, IEEE Transactions on, 39(10), pp. 14821493

[17] Giridhar, A. and Kumar, P., 2006. Distributed clock synchronization over wireless networks: Algorithms and analysis, Decision and Control, 2006 45th IEEE Conference on 2006, IEEE, pp. 4915-4920.

[18] Han, J. and Jeong, D., 2010. A practical implementation of IEEE 15882008 transparent clock for distributed measurement and control systems. Instrumentation and Measurement, IEEE Transactions on, 59(2), pp. 433-439. 